\title{
Assembling and encoding word representations: fMRI subsequent memory effects implicate a role for phonological control
}

\author{
Dav Clark ${ }^{\mathrm{a}}$, Anthony D. Wagner ${ }^{\mathrm{a}, \mathrm{b}, \mathrm{c}, *}$ \\ ${ }^{a}$ Department of Brain and Cognitive Sciences, Massachusetts Institute of Technology, 77 Massachusetts Avenue, Cambridge, MA 01239, USA \\ ${ }^{\mathrm{b}}$ Picower Center for Learning and Memory, Massachusetts Institute of Technology, Cambridge, MA 02139, USA \\ ${ }^{\mathrm{c}}$ Martinos Center for Biomedical Imaging, MGH/MIT/HMS, Charlestown, MA 02129, USA
}

\begin{abstract}
Novel word learning is central to the flexibility inherent in the human language capacity. Word learning may partially depend on long-term memory formation during the assembly of phonological representations from orthographic inputs. In the present study, event-related functional magnetic resonance imaging (fMRI) examined the contributions of phonological control—a component of the verbal working memory system - to phonological assembly and word learning. Subjects were scanned while making syllable decisions about visually presented familiar (English) and novel (pseudo-English and Foreign) words, a task that required retrieval and analysis of existing phonological codes or the assembly and analysis of novel representations. Results revealed that left inferior prefrontal cortex (LIPC) and bilateral parietal cortices were differentially engaged during the processing of novel words, suggesting that this circuit is recruited during phonological assembly. A subsequent memory analysis that examined the relation between fMRI signal and the subject's ability to later remember the words (a measure of effective memory formation) revealed that the magnitude of activation in LIPC, bilateral superior parietal, and left inferior parietal cortices was positively correlated with later memory. Moreover, although the magnitude of the subsequent memory effect in parietal cortex was not significantly affected by word type, this effect was greater in posterior LIPC for novel (pseudo-English) than for familiar (English) words. In the course of subserving the assembly of novel word representations, the phonological (articulatory) control component of the phonological system appears to play a central role in the encoding of novel words into long-term memory.
\end{abstract}

(C) 2002 Published by Elsevier Science Ltd.

Keywords: Phonological loop; Verbal working memory; Episodic encoding; Explicit memory; Visual word recognition

\section{Introduction}

Current understanding of language processing distinguishes a set of component linguistic functions that include, but by no means are limited to, orthographic operations supporting processing of the visual structure of written words and phonological operations supporting processing of the corresponding speech sounds [10]. A central aspect of language acquisition-learning of novel phonological patterns that correspond to new words-may be partially subserved by phonological operations that mediate orthographic-to-phonological translation and on-line storage of the resultant phonological codes [2]. This translation may be supported by the phonological (articulatory) control component of the phonological system [3], which is partially subserved by the posterior extent of the left inferior prefrontal cortex (pLIPC; Brodmann's areas [BA]

\footnotetext{
* Corresponding author. Tel.: +1-617-452-2492; fax: +1-617-258-8654. E-mail address: awagner@psyche.mit.edu (A.D. Wagner).
}

44/6) $[1,29,39]$. By contrast, the resultant phonological representations may be temporarily maintained during verbal processing; on-line storage of speech-related sound patterns has been associated with inferior and superior parietal cortices $(\sim$ BA $40 / 7)$ [16,23,40]. Critically, in the course of assembling and temporarily storing novel phonological codes, the phonological system may contribute to the formation of durable word representations [27].

Initial behavioral and neuropsychological evidence suggests that the phonological processing system may be particularly important for representing novel phonological codes, and, in the process, for encoding these novel representations into long-term memory [2]. For example, when articulatory suppression disrupts the phonological control mechanism of Baddeley's phonological loop, there is an associated impairment in learning of word-nonword pairs but not of word-word pairs [27]. Moreover, longer (multi-syllabic) word lengths-which challenge the phonological control mechanism-negatively affect word-nonword learning but not word-word learning [28]. Finally, neural insult that 
disrupts phonological working memory leads to impaired word-nonword associative learning but leaves word-word learning relatively intact $[4,5]$.

Multiple lines of evidence implicate pLIPC in orthographic-to-phonological translation and analysis. For example, phonological dyslexia - an impairment in the ability to derive phonological representations from orthographic inputs-is often observed in patients with damage to LIPC [20]. Neuroimaging studies have observed pLIPC activation during tasks requiring analysis of phonological representations $[32,34,55]$, such as rhyme and syllable judgments. Moreover, activation in pLIPC has been reported during tasks involving visually presented nonwords [35], with initial evidence suggesting that greater pLIPC activation is observed during phonological processing of novel (e.g. pseudo-English) relative to familiar (e.g. English) words $[32,41]$. Unfamiliar words may differentially require phonological assembly whereby the orthographic inputs are translated into a novel phonological form, whereas retrieval of phonological codes associated with familiar stimuli may be highly facilitated by existing representations, thus minimizing demands on the phonological control process putatively subserved by pLIPC [13].

Recent event-related functional magnetic resonance imaging (fMRI) data also indicate that engagement of the phonological processing system impacts episodic memory formation. For example, the magnitude of pLIPC and bilateral superior parietal activation during rote verbal rehearsal of familiar words was observed to predict whether the words would be later remembered or forgotten [16]. Such subsequent memory effects provide powerful evidence regarding the neural substrates of memory formation as they link neural responses during goal-directed stimulus processing with a behavioral measure of effective encoding (i.e. later remembering) [26,50]. Extant fMRI data, however, do not address whether the role of pLIPC and parietal computations in verbal encoding differs depending on whether pre-existing phonological representations are available. Moreover, although Baddeley et al. [2] have hypothesized that the storage mechanism, rather than the phonological control component, of the phonological system is particularly critical for new word learning, evidence for a differential role of these sub-components is lacking.

The present event-related fMRI experiment examined whether differences in phonological familiarity modulate activation in the pLIPC and parietal regions previously associated with phonological control and storage, respectively, $[1,16,23,29,39,40]$. We adopted a reverse inference logic that assumed that pLIPC activation reflects engagement of phonological control and inferior and superior parietal activation reflects on-line storage. In so doing, we further explored whether the extent of activation in these regions-and thus recruitment of control and storage processes-is differentially associated with later subsequent remembering for novel words. Given prior observations of pLIPC and parietal activation during performance of syl- lable decision tasks [25,32,34], here subjects were scanned while making syllable decisions about familiar (English) and novel (pseudo-English and Foreign) words. For familiar words, this task requires the retrieval and analysis of an existing phonological representation, whereas for novel words, phonological representations first must be assembled based on knowledge about orthographic-to-phonological mappings. The relation between activation during these phonological processing scans and memory formation was indexed through a subsequent memory analysis. This analysis further probed whether the magnitude of the subsequent memory effect differed for novel and familiar words, and, if so, whether there was evidence for such differences to be greater in parietal regions associated with on-line phonological storage or frontal regions associated with phonological assembly and rehearsal. Finally, the differential response of anterior left inferior prefrontal cortex (aLIPC; $\sim \mathrm{BA} 47 / 45$ ) across word types was indexed as the present design permits an assessment of whether this region, which has been previously associated with controlled semantic retrieval $[6,9,19,21,30,36,46]$, is also engaged during phonological processing conditions.

\section{Methods}

\subsection{Participants}

Twenty right-handed, native speakers of English (11 females; ages 18-33 years), with normal or corrected-to-normal vision, received US\$ 50 for participation. Data from two additional participants were excluded, one due to hardware malfunction whereby behavioral responses were not recorded and one due to motion artifact. Informed consent was obtained in a manner approved by the Committee on the Use of Humans as Experimental Subjects at MIT and the Human Research Committee at Massachusetts General Hospital.

\subsection{Stimuli}

The stimuli consisted of 160 words from each of six classes: two- and three-syllable English, pseudo-English, and Foreign words. For a given subject, the English and pseudo-English words were drawn from the same pool of 640 English nouns. For each word in this pool, a pseudo-English word was generated by replacing one of the consonants in the base word with another randomly generated consonant, maintaining pronouncability. Across subjects, each base English word served as both an English and a pseudo-English stimulus. Following Papagno et al. [27], Finnish words served as the Foreign words; these stimuli were obtained from Finnish news sources.

For counterbalancing purposes, each group of 160 words per class was divided into A and B subgroups matched for mean word length (7.4 letters) and, for the English and 
pseudo-English conditions, mean word frequency of the base English word (8.0 occurrences per million [24]). As word length was matched across the two- and three-syllable items, the syllable judgments were necessarily based on phonological, rather than visual, representations.

\subsection{Behavioral procedure}

The experimental session consisted of phonological processing of the three classes of words during four fMRI study phases, followed $20 \mathrm{~min}$ later by a recognition memory test administered in an adjacent behavioral testing room. Brief practice on the phonological task was conducted in the scanner immediately prior to the four study scans.

During each study trial, a single word was visually displayed and subjects indicated, as quickly and accurately as possible, whether the word consisted of two- or threesyllables by pressing one of two response keys under their left hand. Stimuli were presented for $0.8 \mathrm{~s}$, with a $1.2 \mathrm{~s}$ fixation ("+") displayed following stimulus offset (participants had the entire $2 \mathrm{~s}$ to respond). In addition, fixation null events ranging from 2 to $6 \mathrm{~s}$, "jittered" in increments of $2 \mathrm{~s}$, were randomly interleaved between experimental trials as determined by an optimization algorithm [15]. The order of trials within and across scan runs was determined by optimizing the efficiency of the design matrix [15].

For the (non-scanned) subsequent memory test phase, participants performed a surprise old/new recognition task on all studied items and an equal number of distractors. Items were presented in a self-paced fashion in 10 blocks of equal size, with a brief break permitted between blocks. For each item, subjects indicated whether the item was encountered during the fMRI study scans (OLD) or was unstudied (NEW); when responding "OLD", an additional confidence rating was requested ("high" or "moderate"). For each word type, items from each of the four study phases were randomly distributed to each of the 10 blocks of the test phase.

\section{4. fMRI imaging procedure}

Scanning was performed on a 1.5T Siemens Sonata MRI system using a whole-head coil. Functional data were acquired using a gradient-echo echo-planar pulse sequence $(\mathrm{TR}=2 \mathrm{~s}, \mathrm{TE}=40 \mathrm{~ms}, 21$ axial slices, $3.125 \mathrm{~mm} \times$ $3.125 \mathrm{~mm} \times 5 \mathrm{~mm}, 1 \mathrm{~mm}$ inter-slice gap, 190 volume acquisitions per run). High-resolution T1-weighted (MP-RAGE) anatomical images were collected for anatomical visualization. Head motion was restricted using a bite-bar. Visual stimuli were projected via a collimating lens onto a screen, which was viewed through a mirror attached to the head coil.

Data were preprocessed using SPM99 (Wellcome Department of Cognitive Neurology, London). Images were corrected for differences in slice acquisition timing by resampling all slices in time to match the first slice, followed by motion correction across all runs (using since interpolation). Structural and functional data were spatially normal- ized to an EPI template based on the MNI305 stereotactic space [11], an approximation of canonical space [42], using a 12 parameter affine transformation along with a nonlinear transformation using cosine bases functions. Images were resampled into 3-mm cubic voxels and then spatially smoothed with an 8-mm FWHM isotropic Gaussian kernel.

Statistical analysis was performed using the general linear model in SPM99. Correct trials from each condition were modeled using a canonical hemodynamic response, with error trials from all conditions modeled separately. Effects were estimated using a subject-specific fixed-effects model, with session-specific effects and low-frequency signal components treated as confounds. Linear contrasts were used to obtain subject-specific estimates for each effect. These estimates were entered into a second-level analysis treating subjects as a random effect, using a one-sample t-test against a contrast value of zero at each voxel. Given a priori expectations that LIPC and parietal activation would accompany performance of the phonological encoding task, regions were considered reliable to the extent that they consisted of at least five contiguous voxels that exceeded an uncorrected threshold of $P<0.001$. Given the lower power associated with the subsequent memory analysis-due to the splitting of trials into smaller bins based on later memory-as well as these a priori predictions, subsequent memory effects were considered reliable based on the criteria of at least five contiguous voxels exceeding an uncorrected threshold of $P<$ 0.005 .

The group-level voxel-based contrasts were supplemented with region-of-interest (ROI) analyses that further characterized the degree to which the neural responses in functionally defined, and a priori predicted, prefrontal and parietal regions were correlated with subsequent recognition memory. For the ROI analysis, spherical regions of interest were identified by choosing all significant voxels within an $8-\mathrm{mm}$ radius of the chosen maximum identified in the group statistical map. Signal within each ROI was then calculated for each individual subject by selectively averaging the data with respect to peristimulus time for trials in each condition. Peak percent signal change, where the peak was defined by comparing all trial types to baseline, for each condition was entered into a mixed-effects analysis of variance (ANOVA) that treated subsequent memory outcome (high confidence remembered/forgotten) and word type (word/pseudo-word) as repeated measures, with subjects treated as a random effect. Prior studies have shown that, when sufficient power is present, fMRI correlates of subsequent memory are largest when comparing high confidence hits to forgotten trials (e.g. [54]).

Due to the near-chance level of subsequent memory performance for the Foreign words (see Section 3), this condition was excluded from the subsequent memory analysis as many of the high confidence remembered trials for Foreign words likely reflect guesses. Exclusion of the Foreign words from this analysis was also necessitated because, for 9 of the 20 subjects, an insufficient number of trials were available 
for a given cell to provide adequate signal to noise (using a criterion of at least 12 items/cell). Thus, the subsequent memory analysis was performed exclusively for the English and pseudo-English conditions. For 3 of the 20 subjects, an insufficient number of trials were available for a given cell in the English or pseudo-English conditions, and thus these three subjects were excluded from the subsequent memory analysis.

\section{Results}

\subsection{Behavioral results}

Behavioral effects were considered reliable at an alpha level of 0.05 . Accuracy on the phonological judgment task was high for all word types (Table 1), though performance significantly differed across word type $(F(2,38)=34.81)$. Accuracy was greater for English relative to pseudo-English words $(F(1,19)=24.91)$, with the latter being greater relative to Foreign words $(F(1,19)=10.86)$.

To control for differences in accuracy between word classes, only correct trials were included in all subsequent behavioral and fMRI analyses. Response latency differed across word type $(F(2,38)=41.36)$, being significantly faster for English as compared to pseudo-English $(F(1$, $19)=46.09)$ and Foreign $(F(1,19)=74.58)$ words. There was a trend for slower responses to Foreign words than to pseudo-English words $(F(1,19)=3.41, P<0.08)$.

With respect to subsequent memory performance, hit and false alarm rates on the recognition memory test are displayed in Table 1. Corrected recognition (pHit - pFalse alarm), computed both when collapsing across confidence levels ("high" and "moderate") and separately for "high confidence" responses, differed across word type (overall: $F(2,38)=41.10$; high confidence: $(F(2,38)$ $=29.36)$. Subsequent memory was superior for English than pseudo-English words (overall: $F(1,19)=5.58$; high con-

Table 1

Accuracy and reaction time (in ms) on the syllable decision task, and subsequent hit rates to studied and false alarm rates to unstudied recognition probes

\begin{tabular}{llllll}
\hline & \multicolumn{2}{c}{ Syllable judgments } & & \multicolumn{2}{c}{ Subsequent memory } \\
\cline { 2 - 3 } Condition & Accuracy & RT & & "HC-Old" & "MC-Old" \\
\hline English & & & & \\
$\quad$ Studied & $0.91(0.01)$ & $855(47.8)$ & $0.32(0.04)$ & $0.19(0.02)$ \\
$\quad$ Unstudied & & & $0.08(0.02)$ & $0.16(0.03)$ \\
$\begin{array}{l}\text { Pseudo-English } \\
\quad\end{array}$ & & & & \\
$\quad \begin{array}{l}\text { Studied } \\
\quad \text { Unstudied }\end{array}$ & $0.84(0.02)$ & $928(53.8)$ & $0.22(0.03)$ & $0.23(0.03)$ \\
$\quad$ & & & $0.05(0.01)$ & $0.17(0.03)$ \\
Foreign & & & & \\
$\quad \begin{array}{l}\text { Studied } \\
\quad \text { Unstudied }\end{array}$ & $0.79(0.02)$ & $948(53.6)$ & $0.15(0.02)$ & $0.38(0.03)$ \\
\hline
\end{tabular}

Note-"HC-Old": high confidence-old; "MC-Old": moderate confidenceold; RT: reaction time (standard error of mean). fidence: $F(1,19)=8.38$ ), with the latter being superior to memory for Foreign words (overall: $F(1,19)=40.94$; high confidence: $F(1,19)=22.07)$. There was no relation between subsequent memory outcome and encoding response latency $(F(2,38)=1.50, P>0.20)$-that is, the response times for the syllable judgment on words later remembered with high confidence (English, 850; pseudo-English, 927; Foreign, $918 \mathrm{~ms}$ ), later remembered with moderate confidence (English, 867; pseudo-English, 923; Foreign, $954 \mathrm{~ms}$ ), and later forgotten (English, 849; pseudo-English, 936; Foreign, $933 \mathrm{~ms}$ ) were comparable. The Type $\times$ Memory interaction did not approach significance $(F<1)$. Similarly, there was no relation between subsequent memory outcome and word frequency $(F<1)$ : high confidence remembered (English, 7.7; pseudo-English, 8.7), low confidence remembered (English, 7.6; pseudo-English, 8.0), and forgotten (English, 8.0; pseudo-English, 7.9). "Word frequency" for pseudo-English items corresponded to the frequency of the base English word from which the pseudo-English word was generated. The Type $\times$ Memory interaction was not reliable $(F(2,38)=1.32, P>0.25)$.

The behavioral measures of subsequent recognition were used to conduct a subsequent memory analysis on the fMRI encoding data. As discussed above, due to the near-chance level of memory for the Foreign words, this word class was excluded from the subsequent memory analysis.

\subsection{Imaging results: task and stimulus effects}

Voxel-based statistical analyses revealed that performance of the syllable judgment task - collapsed across word typerelative to the fixation baseline elicited activation in occipital, parietal, and frontal regions (Fig. 1). These regions included the posterior extent of the left inferior prefrontal cortex (pLIPC; $\sim$ BA 44/6) and bilateral inferior and superior parietal ( $\sim \mathrm{BA} 40 / 7)$ cortices previously associated with phonological processing of visually presented words and pseudo-words $[19,25,32,34,41]$, as well as with phonological working memory $[1,14,23,29,33,40,51]$. Thus, the syllable judgment task elicited involvement of prefrontal regions associated with phonological control and parietal regions associated with the on-line representation of phonological codes. In addition, a response during the phonological task was observed in the most anterior extent of the left inferior prefrontal cortex (aLIPC), bordering on frontopolar cortex ( $\sim$ BA 47/46/10; Fig. 1). As considered in the Discussion, this focus fell just anterior to previously observed foci associated with controlled semantic retrieval.

The above pattern of frontal and parietal activation was also observed when separately contrasting each of the word types to baseline, with the magnitude of activation appearing to be particularly robust for pseudo-English words and more modest for English words. To further assess differences in phonological processing across word types, direct voxel-based comparisons were performed (Fig. 1; Table 2). Relative to English words, both pseudo-English and For- 

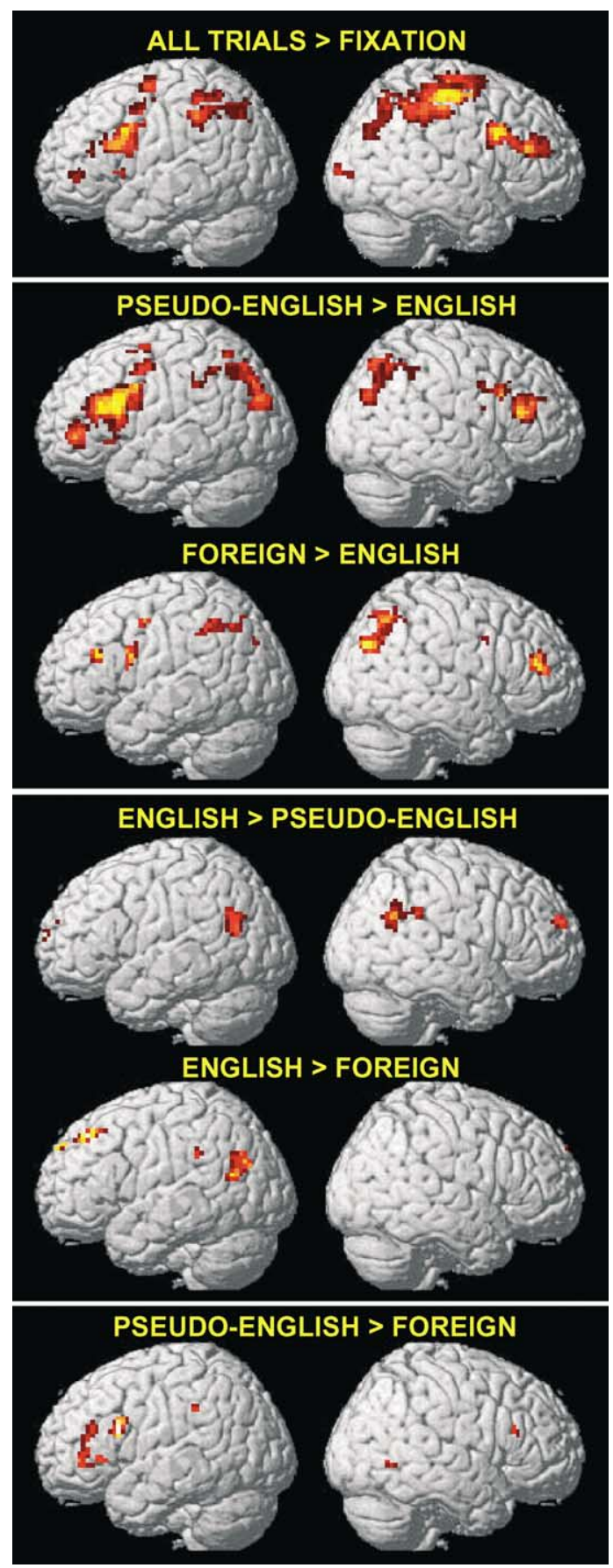

Fig. 1. Functional activation maps revealed prefrontal and parietal cortices associated with phonological task performance (All Trials > Fixation), as well as their differential engagement across word types. Bilateral inferior prefrontal and parietal cortices were more active during the processing of unfamiliar (pseudo-English and Foreign) relative to familiar (English) words. A statistical threshold of $P<0.001$ and cluster constraint of five voxels were applied.

eign words elicited greater activation in LIPC and bilateral parietal cortices, as well in right inferior prefrontal cortex (RIPC). Direct comparison of pseudo-English to Foreign trials revealed that the frontal foci as well as a modest left inferior parietal region were engaged to a greater extent dur- ing the former condition. This was the case even though there was a trend for longer reaction times to Foreign words, which is consistent with prior observations indicating that lateral frontal activation does not track generalized task difficulty (as indexed by reaction time) [18,32]. By contrast, greater activation was observed during English relative to pseudo-English and Foreign trials in bilateral inferior parietal/angular gyrus, posterior cingulate, and medial frontopolar cortices. Collectively, these direct comparisons indicate that the pLIPC and parietal regions associated with phonological control and representation are differentially engaged during the phonological assembly and analysis of novel (pseudo-English and Foreign) relative to familiar (English) words.

\subsection{Imaging results: subsequent memory effects}

Voxel-based analyses revealed greater activation in LIPC, RIPC, and bilateral superior parietal cortices during the encoding of words (collapsed across pseudo-English and English) that were subsequently recognized with high confidence relative to those later forgotten (Fig. 2; Table 3). These foci included multiple pLIPC peaks that fell near the peak $(-39,9,30)$ observed by Davachi et al. [16] to correlate with later memory following rote phonological rehearsal. The left $(-27,-72,54)$ and right $(30,-75,51)$ superior parietal foci fell just posterior to those of Davachi et al. ( $-24,-57,45$ and 30, $-63,45$, respectively). These across-study similarities suggest that the phonological system that supports verbal working memory also subserves the episodic encoding of word representations during phonological assembly and analysis. Although the present task, as well as that of Davachi et al., nominally required phonological, rather than semantic processing, both studies observed a subsequent memory effect in aLIPC (present: $-45,36$, -9; Davachi et al.: $-45,30,-9)$, with the present study observing a second aLIPC peak that bordered frontopolar cortex $(-48,42,3)$.

Region-of-interest analyses conducted on the foci identified in the subsequent memory analysis revealed a reliable Type (English/pseudo-English) $\times$ Memory (remembered/ forgotten) interaction in pLIPC $(-45,24,18: F(1,16)$ $=5.84, P<0.05 ;-54,18,21: F=3.69, P<0.08)$, as well as a trend for such an interaction in the most rostral aLIPC/frontopolar focus $(-48,42,3: F=4.22, P<0.06)$. These interactions reflected a greater subsequent memory effect for the novel pseudo-English words than for the known English words in each region (Fig. 3, A-C). Although larger for pseudo-English words, the subsequent memory effect was reliable for English words in the more caudal pLIPC focus $(-54,18,21: t(16)=2.08, P=0.05)$, was unreliable in the more rostral pLIPC focus $(-45,24,18: t=1.04$, $P>0.30$ ), and approached significance in the rostral aLIPC/frontopolar region $(t=1.90, P<0.08)$. By contrast, the Type $\times$ Memory interaction was not reliable in the other aLIPC focus $(-45,36,-9: P>0.90)$, which demonstrated 
Table 2

Regions demonstrating a differential response during phonological processing of English, pseudo-English, and Foreign words

\begin{tabular}{|c|c|c|c|c|c|}
\hline \multirow[t]{2}{*}{ Region } & \multicolumn{3}{|c|}{ MNI coordinates } & \multirow[t]{2}{*}{$\sim \mathrm{BAs}$} & \multirow{2}{*}{$\begin{array}{l}\text { Peak } Z \text { score } \\
\text { (number of voxels) }\end{array}$} \\
\hline & $x$ & $y$ & $z$ & & \\
\hline \multicolumn{6}{|l|}{ Pseudo-English > English } \\
\hline L middle/inferior frontal & -51 & 33 & 21 & $46 / 45$ & $4.97(515)$ \\
\hline $\mathrm{L}$ inferior frontal & -51 & 15 & 21 & 44 & 4.50 \\
\hline $\mathrm{L}$ middle/frontopolar & -39 & 48 & 3 & $10 / 46$ & 4.41 \\
\hline $\mathrm{L}$ precentral/inferior frontal & -45 & 3 & 27 & $6 / 44$ & 4.16 \\
\hline L middle frontal & -51 & 3 & 45 & 6 & $4.83(31)$ \\
\hline $\mathrm{L}$ intraparietal & -24 & -69 & 33 & $7 / 19$ & $4.78(439)$ \\
\hline L superior parietal & -27 & -51 & 45 & $7 / 40$ & 4.56 \\
\hline $\mathrm{L}$ intraparietal & -21 & -69 & 48 & 7 & 4.51 \\
\hline $\mathrm{L}$ middle occipital & -30 & -81 & 24 & 19 & 4.20 \\
\hline $\mathrm{R}$ intraparietal & 30 & -66 & 39 & $7 / 19$ & 4.49 (334) \\
\hline R superior/inferior parietal & 33 & -60 & 48 & $7 / 40$ & 4.47 \\
\hline $\mathrm{R}$ supramarginal/inferior parietal & 30 & -57 & 30 & $40 / 7$ & 4.37 \\
\hline $\mathrm{R}$ inferior frontal & 45 & 30 & 15 & 45 & $4.10(158)$ \\
\hline $\mathrm{R}$ middle/inferior frontal & 57 & 18 & 36 & $9 / 44$ & 3.53 \\
\hline $\mathrm{R}$ precentral & 42 & 0 & 33 & 6 & 3.52 \\
\hline Medial superior frontal & -3 & 12 & 51 & $6 / 8$ & $3.97(20)$ \\
\hline $\mathrm{L}$ superior/middle frontal & -30 & -3 & 63 & 6 & $3.69(15)$ \\
\hline L superior frontal & -24 & 6 & 60 & 6 & 3.55 \\
\hline Medial superior frontal & 0 & 6 & 60 & 6 & $3.54(5)$ \\
\hline L superior parietal & -30 & -60 & 57 & 7 & $3.50(13)$ \\
\hline L superior frontal & -18 & 3 & 51 & 6 & $3.48(5)$ \\
\hline $\mathrm{R}$ lingual/fusiform & 18 & -63 & 0 & $18 / 19$ & $3.45(7)$ \\
\hline $\mathrm{R}$ inferior frontal & 42 & 9 & 18 & 44 & $3.42(15)$ \\
\hline \multicolumn{6}{|l|}{ Foreign $>$ English } \\
\hline $\mathrm{R}$ superior/inferior parietal & 30 & -60 & 45 & $7 / 40$ & $4.90(156)$ \\
\hline $\mathrm{R}$ intraparietal/inferior parietal & 27 & -60 & 33 & $7 / 40$ & 4.08 \\
\hline $\mathrm{R}$ inferior/superior parietal & 36 & -48 & 45 & $40 / 7$ & 3.49 \\
\hline $\mathrm{R}$ middle/superior occipital & 30 & -81 & 36 & 19 & 3.48 \\
\hline $\mathrm{L}$ inferior parietal & -39 & -45 & 39 & 40 & $4.36(156)$ \\
\hline L superior/inferior parietal & -36 & -51 & 45 & $7 / 40$ & 4.11 \\
\hline L superior/inferior parietal & -30 & -63 & 42 & $7 / 40$ & 3.82 \\
\hline $\mathrm{L}$ precentral/middle frontal & -48 & -3 & 48 & $4 / 6$ & $3.88(10)$ \\
\hline $\mathrm{L}$ inferior frontal & -45 & 6 & 27 & $44 / 6$ & $3.85(38)$ \\
\hline $\mathrm{L}$ inferior frontal & -30 & 6 & 27 & $44 / 6$ & 3.67 \\
\hline $\mathrm{L}$ inferior frontal & -48 & 9 & 15 & 44 & 3.53 \\
\hline $\mathrm{L}$ inferior/middle frontal & -45 & 30 & 24 & $45 / 46$ & $3.78(12)$ \\
\hline $\mathrm{R}$ middle frontal & 51 & 39 & 21 & $46 / 9$ & $3.67(23)$ \\
\hline $\mathrm{R}$ middle frontal & 39 & 42 & 24 & $46 / 9$ & 3.57 \\
\hline $\mathrm{R}$ middle/frontopolar & 48 & 48 & 15 & $10 / 46$ & 3.48 \\
\hline \multicolumn{6}{|l|}{ English > pseudo-English } \\
\hline $\mathrm{R}$ middle/frontopolar & 24 & 54 & 24 & $9 / 10$ & $4.77(20)$ \\
\hline Medial orbital frontal & 0 & 54 & -9 & 10 & $4.73(163)$ \\
\hline Medial frontal & -6 & 57 & 3 & 10 & 4.45 \\
\hline Medial frontal & -3 & 60 & 21 & 9 & 4.24 \\
\hline $\mathrm{R}$ superior temporal & 57 & -60 & 27 & 39 & $4.55(59)$ \\
\hline $\mathrm{R}$ inferior parietal & 66 & -39 & 30 & 40 & 4.05 \\
\hline $\mathrm{L}$ middle temporal/angular & -51 & -66 & 18 & 39 & $4.44(53)$ \\
\hline $\mathrm{L}$ inferior parietal/angular & -45 & -69 & 30 & $39 / 19$ & 3.39 \\
\hline Posterior cingulate & -9 & -48 & 30 & 31 & $4.39(44)$ \\
\hline Posterior cingulate & 0 & -18 & 39 & 23 & $3.96(31)$ \\
\hline
\end{tabular}


Table 2 (Continued)

\begin{tabular}{|c|c|c|c|c|c|}
\hline \multirow[t]{2}{*}{ Region } & \multicolumn{3}{|c|}{ MNI coordinates } & \multirow[t]{2}{*}{$\sim$ BAs } & \multirow{2}{*}{$\begin{array}{l}\text { Peak } Z \text { score } \\
\text { (number of voxels) }\end{array}$} \\
\hline & $x$ & $y$ & $z$ & & \\
\hline \multicolumn{6}{|l|}{ English > Foreign } \\
\hline L inferior parietal/angular & -45 & -72 & 27 & $39 / 19$ & $4.14(58)$ \\
\hline L middle temporal/angular & -48 & -63 & 18 & 39 & 3.86 \\
\hline L superior/middle frontal & -21 & 36 & 45 & 8 & $3.88(40)$ \\
\hline L superior frontal & -15 & 57 & 36 & 9 & $3.84(16)$ \\
\hline Posterior cingulate/precuneus & -9 & -42 & 42 & $31 / 7$ & $3.68(12)$ \\
\hline Posterior cingulate/precuneus & -3 & -51 & 42 & $31 / 7$ & 3.35 \\
\hline Medial frontal & -6 & 54 & 21 & 9 & $3.62(24)$ \\
\hline Medial frontal & -3 & 60 & 6 & 10 & 3.49 \\
\hline $\mathrm{L}$ inferior parietal & -60 & -39 & 33 & 40 & $3.56(6)$ \\
\hline \multicolumn{6}{|l|}{ Pseudo-English > Foreign } \\
\hline L inferior frontal & -48 & 30 & 3 & $45 / 47$ & $3.86(10)$ \\
\hline $\mathrm{L}$ inferior frontal & -42 & 12 & 24 & 44 & $3.81(60)$ \\
\hline $\mathrm{L}$ inferior frontal & -51 & 15 & 27 & $44 / 9$ & 3.76 \\
\hline $\mathrm{L}$ inferior frontal & -57 & 18 & 21 & $44 / 9$ & 3.60 \\
\hline L inferior frontal & -39 & 39 & -3 & $47 / 10$ & $3.74(41)$ \\
\hline $\mathrm{L}$ middle/inferior frontal & -51 & 33 & 24 & $46 / 45$ & 3.61 \\
\hline L inferior frontal & -48 & 39 & 6 & $45 / 47 / 46$ & 3.59 \\
\hline $\mathrm{L}$ inferior parietal & -54 & -36 & 36 & 40 & $3.37(6)$ \\
\hline $\mathrm{R}$ fusiform & 45 & -60 & -3 & 37 & $3.32(6)$ \\
\hline $\mathrm{R}$ inferior/middle temporal & 54 & -63 & 0 & 37 & 3.16 \\
\hline $\mathrm{R}$ inferior/middle frontal & 48 & 24 & 21 & $45 / 9$ & $3.31(6)$ \\
\hline
\end{tabular}

reliable subsequent memory effects for both pseudo-English and English words (Fig. 3, D). This aLIPC focus fell closer to the region frequently observed during controlled semantic retrieval. Finally, the Type $\times$ Memory interaction was not significant in RIPC, which showed reliable subsequent memory effects for both words types, nor was it significant in superior parietal cortices $(P>0.10)$. However, there was a quantitatively larger subsequent memory effect for pseudo-English words in both left and right superior parietal cortex (Fig. 3, E-F); the memory effect for English words was not reliable in the former region $(t=1.31, P>0.20)$, but was significant in the latter $(t=2.53, P<0.05)$.

A striking characteristic of the voxel-based analysis is the absence of subsequent memory effects in the inferior parietal regions associated with phonological task performance (Fig. 1). To further explore whether activation in these a priori predicted inferior parietal regions, as well as in frontal foci, was correlated with subsequent memory, additional ROI analyses were conducted on the regions identified as associated with phonological task performance (Fig. 1; All Trials > Fixation). Moreover, to the extent that pLIPC and/or inferior parietal cortical computations are particularly relevant to the assembly of novel phonological representations and their encoding into memory, one would expect activation to correlate more strongly with subsequent memory for pseudo-English than for English words. While a Type $\times$ Memory interaction was borne out in pLIPC $(-36$,
15, 17: $F(1,16)=4.87, P<0.05 ;-54,21,21: F=3.91$, $P<0.07 ;-51,9,18: F=3.42, P<0.09$ ), this critical interaction was not reliable in right nor left inferior parietal cortices (all $P>0.10$; Fig. 3). A main effect of subsequent memory, however, was observed in left, but not right, parietal cortex $(-27,-69,30: F(1,16)=8.56, P<0.01 ;-24$, $-60,45: F(1,16)=3.31, P<0.09 ; 33,-69,33$ and 33 , $-51,48: P>0.36)$. Importantly, the triple interaction of region $(\mathrm{pLIPC} /$ left parietal) $\times$ Type $\times$ Memory was significant, indicating that the differential encoding response to unfamiliar relative to known words was greater in pLIPC than in left inferior parietal cortex $(F(1,16)=5.00, P<0.05)$.

\subsection{Imaging results: subsequent forgetting effects}

Voxel-based analyses also revealed negative correlates of subsequent memory (Fig. 2; Table 3) - that is, regions in which greater activation during encoding was associated with later forgetting [17,25,47]. Although not fully understood at present [25,47], such subsequent forgetting effects may reflect the diversion of attentional resources to cognitive operations that are ineffective for later remembering. Importantly, the present study revealed negative correlates of remembering in right middle and superior frontal, medial parietal/precuneus, posterior cingulate, and right inferior parietal regions that resemble those observed in previous reports $[25,47]$. The magnitude of the responses 


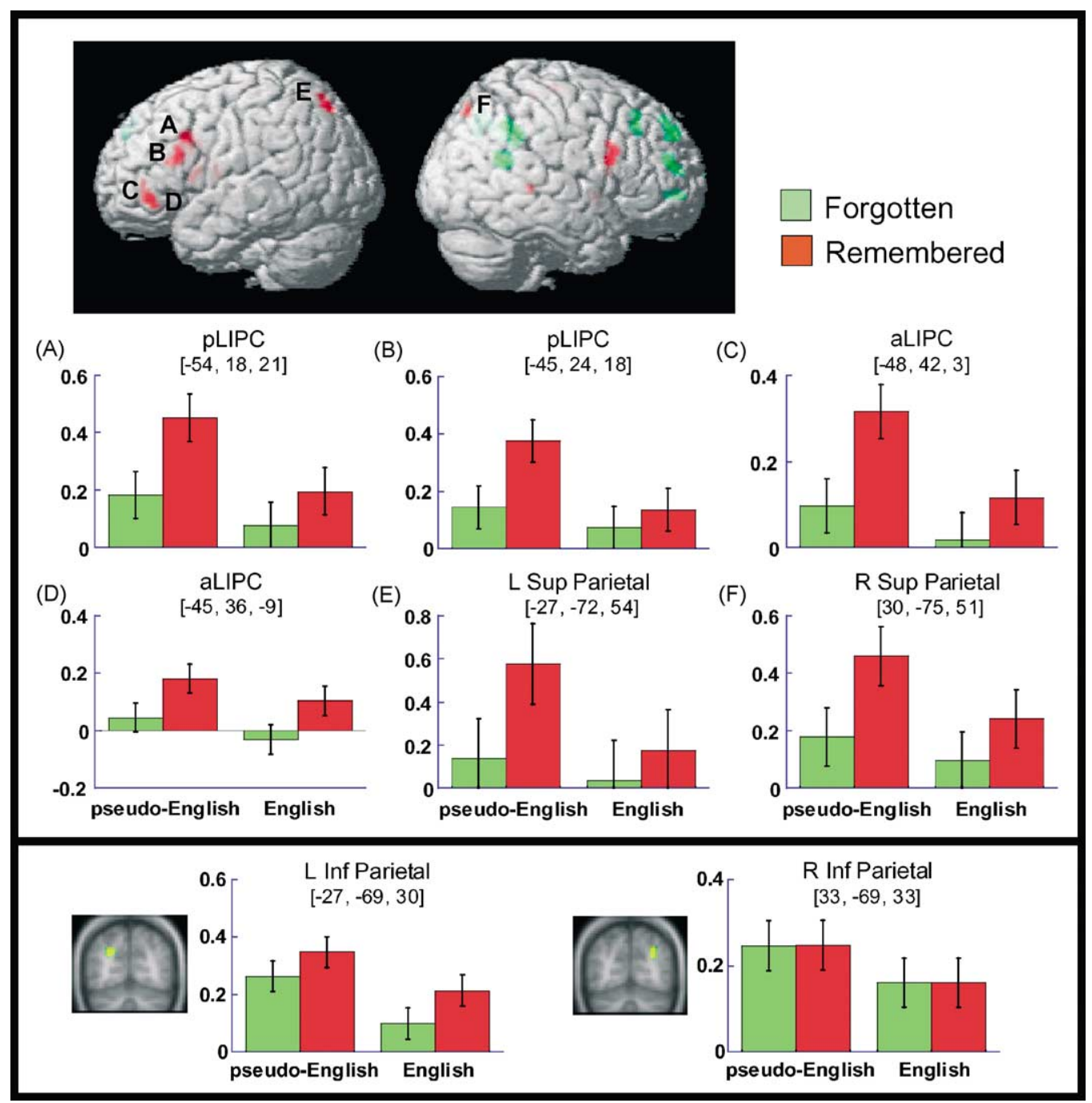

Fig. 2. Activation in frontal and parietal regions demonstrated positive and negative correlations with subsequent memory performance. In the upper panel, the functional map depicts regions that demonstrated greater activation during words later remembered (collapsed across English and pseudo-English) relative to those later forgotten (red), as well as regions demonstrating the reverse pattern (green). ROI graphs depict the peak percent signal change in frontal and parietal regions positively correlated with subsequent remembering. In the lower panel, data are rendered for the inferior parietal ROIs identified when comparing all phonological processing trials to the fixation baseline. Left, but not right, inferior parietal cortex demonstrated a subsequent memory effect for pseudo-English and English words. Error bars depict the 95\% confidence interval for the Memory $\times$ Type interaction.

in these regions consistently fell below the fixation baseline, with a more modest deactivation being associated with later forgetting. Further investigation is required to more fully characterize the nature of the processes subserved by these regions and why they are associated with ineffective encoding.

\section{5. aLIPC activation during phonological processing}

The objective of the present study was to explore the contributions of pLIPC and bilateral inferior and superior parietal mechanisms to phonological assembly and word learning. However, the fMRI outcomes also bear on current debates regarding the nature of the mechanisms subserved by aLIPC. As noted above, an unexpected aLIPC response was observed during performance of the phonological processing task, with the magnitude of activation being greater during pseudo-English relative to English word processing. Prior studies indicate that aLIPC may be differentially engaged during semantic relative to phonological analysis $[25,32,34]$, with some theorists positing a role for this region in controlled access to long-term semantic representations $[6,9,19,21,30,36,46]$. The presently observed response, albeit in a more anterior (bordering on frontopolar) region to that often observed in semantic retrieval studies, raises the possibility that aLIPC contributions may not be restricted to controlled semantic retrieval. Indeed, although some prior studies of phonological analysis of pseudo-English words have failed to observe aLIPC activation relative to nonsemantic baseline conditions (e.g. [32]), others have 
Table 3

Neocortical regions demonstrating positive or negative associations with subsequent memory performance (collapsed across English and pseudo-English words)

\begin{tabular}{|c|c|c|c|c|c|}
\hline \multirow[t]{2}{*}{ Region } & \multicolumn{3}{|c|}{ MNI coordinates } & \multirow[t]{2}{*}{$\sim$ BAs } & \multirow{2}{*}{$\begin{array}{l}\text { Peak } Z \text { score } \\
\text { (number of voxels) }\end{array}$} \\
\hline & $x$ & $y$ & $z$ & & \\
\hline \multicolumn{6}{|l|}{ Remembered $>$ forgotten } \\
\hline $\mathrm{L}$ inferior frontal & -45 & 36 & -9 & $47 / 45$ & $5.02(21)$ \\
\hline $\mathrm{L}$ inferior frontal/frontopolar & -48 & 42 & 3 & $47 / 46 / 10$ & 3.72 \\
\hline $\mathrm{R}$ inferior frontal & 57 & 12 & 18 & 44 & $4.19(23)$ \\
\hline $\mathrm{R}$ inferior frontal & 54 & 12 & 27 & $44 / 9$ & 3.36 \\
\hline Medial frontal/precentral & 9 & -24 & 63 & 4 & $4.09(7)$ \\
\hline $\mathrm{L}$ middle/inferior frontal & -45 & 15 & 36 & $9 / 44$ & $3.92(49)$ \\
\hline $\mathrm{L}$ inferior frontal & -45 & 24 & 18 & 45 & 3.80 \\
\hline $\mathrm{L}$ inferior frontal & -54 & 18 & 21 & $44 / 45$ & 3.47 \\
\hline $\mathrm{L}$ inferior frontal & -39 & 12 & 27 & $44 / 9$ & 3.45 \\
\hline $\mathrm{R}$ frontal operculum/anterior insula & 36 & 12 & 15 & 44 & $3.87(6)$ \\
\hline Paracentral/cingulate & 9 & -33 & 57 & $4 / 31$ & $3.83(5)$ \\
\hline L superior parietal & -27 & -72 & 54 & 7 & $3.65(19)$ \\
\hline $\mathrm{R}$ superior parietal & 30 & -75 & 51 & 7 & $3.41(6)$ \\
\hline $\mathrm{L}$ frontal operculum/anterior insula & -42 & 9 & 12 & 44 & $3.40(9)$ \\
\hline $\mathrm{R}$ middle temporal & 57 & -36 & 0 & 21 & $3.35(5)$ \\
\hline \multicolumn{6}{|l|}{ Forgotten $>$ Remembered } \\
\hline $\mathrm{R}$ middle temporal/angular & 48 & -57 & 18 & 39 & $4.43(28)$ \\
\hline Posterior cingulate & 0 & -24 & 39 & 23 & $4.24(47)$ \\
\hline Posterior cingulate & 9 & -30 & 36 & 23 & 3.99 \\
\hline Medial parietal/precuneus & 9 & -66 & 45 & 7 & $4.02(37)$ \\
\hline Posterior cingulate/precuneus & 6 & -54 & 33 & $31 / 7$ & 3.90 \\
\hline Medial parietal/precuneus & 9 & -60 & 39 & $31 / 7$ & 3.78 \\
\hline $\mathrm{R}$ superior frontal & 27 & 54 & -3 & 10 & $3.83(12)$ \\
\hline $\mathrm{R}$ middle frontal & 42 & 48 & 21 & $46 / 10$ & $3.81(12)$ \\
\hline $\mathrm{R}$ superior frontal & 39 & 57 & 12 & 10 & 3.05 \\
\hline $\mathrm{R}$ middle frontal & 39 & 30 & 39 & $9 / 46$ & $3.67(10)$ \\
\hline $\mathrm{R}$ superior frontal & 24 & 51 & 36 & 9 & $3.58(18)$ \\
\hline $\mathrm{R}$ superior frontal & 27 & 45 & 45 & 9 & 3.42 \\
\hline Medial orbital frontal/cingulate & 6 & 36 & -6 & $10 / 32$ & $3.57(7)$ \\
\hline $\mathrm{R}$ inferior parietal & 60 & -45 & 36 & 40 & $3.52(12)$ \\
\hline $\mathrm{R}$ inferior parietal & 51 & -51 & 39 & 40 & $3.49(10)$ \\
\hline
\end{tabular}

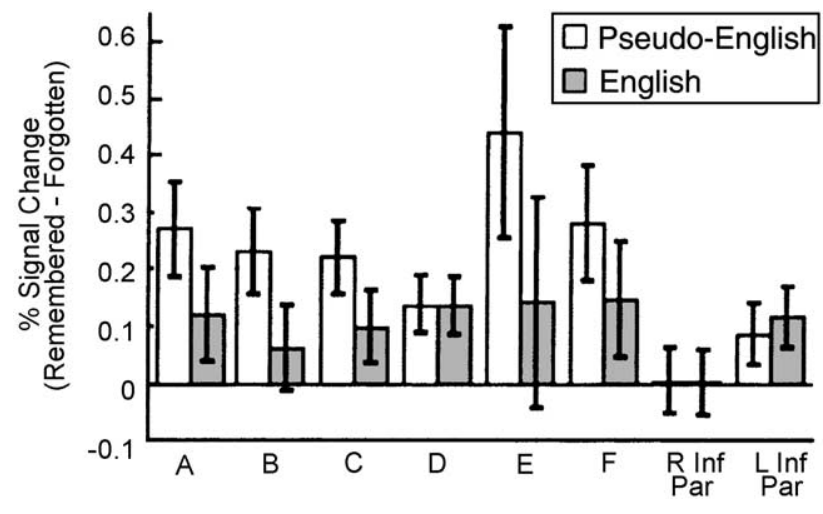

Fig. 3. Magnitude of the subsequent memory effect is plotted for each of the frontal and parietal regions characterized in Fig. 2. Plotted is the difference in peak signal change during the encoding of subsequently remembered and subsequently forgotten words. Error bars depict the $95 \%$ confidence interval for the Memory $\times$ Type interaction. noted increased aLIPC activation during the processing of pseudo-words relative to familiar words (e.g. [41]); the present findings extend these latter results.

A recent study by Gold and Buckner [22] also observed aLIPC activation during phonological decisions about pseudo-words relative to a letter judgment task, although activation in this region was even greater during semantic decisions about English words. These authors assessed the degree to which the various classes of stimuli constrain the construction of task-relevant representations, which was operationalized as between-subject behavioral agreement (or response consensus) on each decision task. Intriguingly, they observed that the magnitude of aLIPC activation across their various stimulus/task conditions was linearly related to the degree of response consensus for the various classes of items/tasks; the lower the response agreement the greater the magnitude of aLIPC activation. Inspired by Gold's analysis [22], we conducted a similar item-analysis 


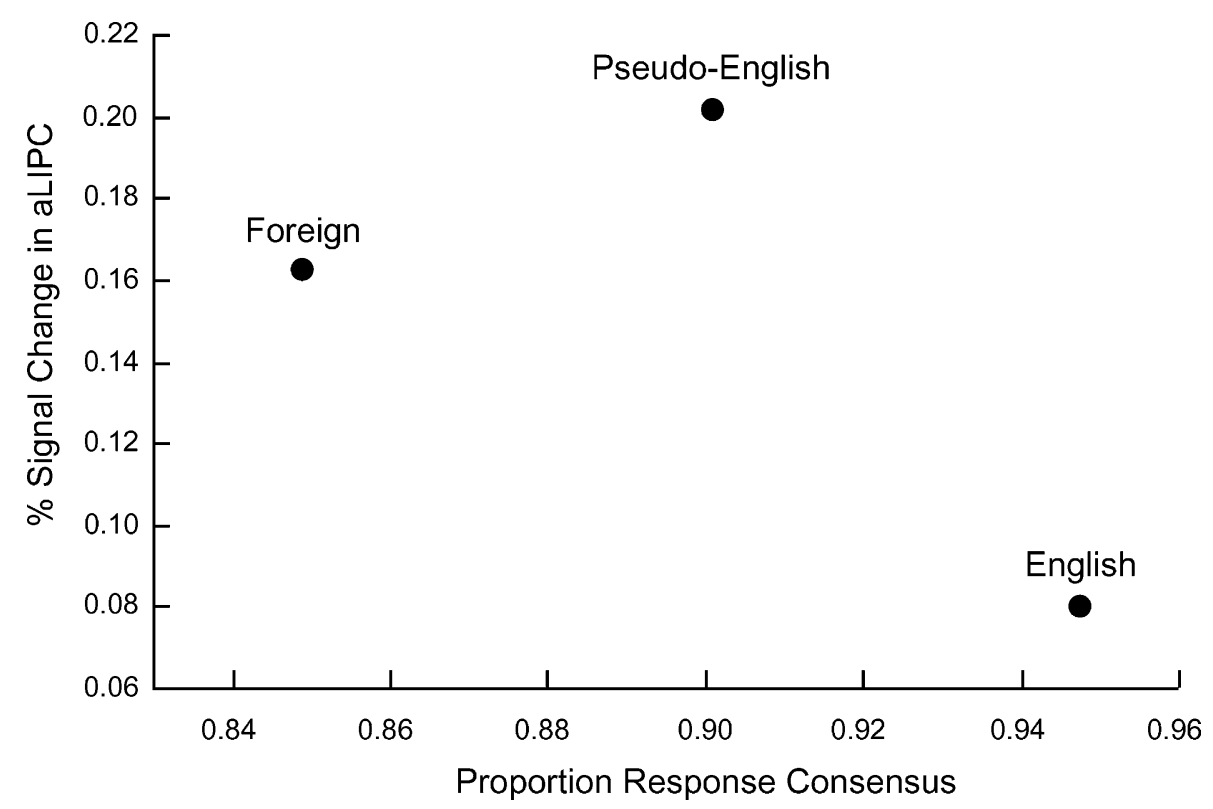

Fig. 4. Between-subject response consensus for each word type is plotted against the magnitude of activation in aLIPC during phonological analysis. Although aLIPC activation was greater for unfamiliar (pseudo-English and Foreign) relative to familiar (English) words, the stimuli that yielded the lowest response consensus did not yield the greatest response in aLIPC (cf. [22]).

to determine response consensus on the syllable judgment task for each word type. Consensus reliably differed across word type $(F(2,1595)=61.03)$, declining from English $(0.95)$ to pseudo-English $(0.90 ; t(1164)=6.39)$, and from the latter to Foreign $(0.85)$ words $(t(664)=5.28)$. We next examined the relation between aLIPC activation and response consensus by plotting these measures against each other (Fig. 4). As can be seen, in contrast to Gold and Buckner's findings [22], aLIPC activation did not linearly increase with decreasing consensus; rather, the pattern resembled an inverted-U. The theoretical implications of this pattern are considered below.

\section{Discussion}

The present experiment assessed the response of pLIPC and bilateral parietal cortices during phonological analysis of novel (pseudo-English and Foreign) and known (English) word forms, and the relation between the magnitude of activation during phonological analysis and subsequent memory performance. The fMRI data revealed four important outcomes regarding the neural underpinnings of phonological assembly and word learning. First, the magnitude of activation in pLIPC and bilateral parietal regions previously associated with phonological control and on-line phonological representation, respectively, was greater during the processing of words that lack an existing representation in memory relative to known words. Second, the magnitude of activation in pLIPC, aLIPC, bilateral superior parietal, and left inferior parietal cortices during phonological analysis was positively correlated with later memory for the words. Third, the magnitude of this subsequent memory effect in pLIPC was greater for novel (pseudo-English) than for known (English) words, whereas the subsequent memory effect in left inferior parietal cortex was comparable across word types. Finally, activation in aLIPC, a region previously associated with controlled semantic retrieval, was greater during phonological analysis of unfamiliar relative to familiar words.

Extensive behavioral data indicate that the phonological (articulatory) control component of the phonological processing system, which has been associated with pLIPC $[1,12,29]$, plays a central role in the transformation of visual word forms to phonological codes [3]. Phonological analysis of visually presented unfamiliar, relative to familiar, words has been posited to differentially require the assembly of novel phonological representations [13], with on-line analysis of the resulting codes requiring maintenance or short-term storage of the newly assembled representations. Differences in control and storage demands across unknown and known word forms is thought to emerge because processing of known words may rely in part upon the retrieval of pre-existing, fully formed representations, whereas the representations of unknown words must be constructed based on knowledge about orthographic-to-phonological mappings. Consistent with this perspective, the present study revealed greater activation in pLIPC ( $\sim$ BA $44 / 6)$ and bilateral parietal cortices $(\sim \mathrm{BA} 7 / 40)$ during the processing of pseudo-English and Foreign words relative to that of familiar English words [32,41].

The present study further assessed the relation between behavioral measures of effective long-term memory formation and fMRI measures of activation in the pLIPC and parietal structures subserving phonological assembly and 
on-line representation. A prior fMRI study of the encoding of known (English) words observed reliable correlates of subsequent memory following phonological analysis (syllable judgments) in bilateral superior parietal cortices, but not in LIPC [25]. By contrast, in a study investigating rote rehearsal of English word triplets, subsequent memory for known words was again observed to correlate with superior parietal activation, with additional correlates observed in pLIPC [16]. The present data extend and further clarify these initial findings. First, consistent with Davachi et al. [16], there was a correlation between subsequent memory outcome following phonological processing of English words and the magnitude of encoding-phase activation in the most caudal portion of $\mathrm{pLIPC}$ and in right superior parietal cortex (Fig. 3). These data indicate that, all else being equal, increased recruitment of phonological control and storage processes is associated with more effective memory formation. Second, the relation between activation in these regions and later remembering was extended to memory for words that lack pre-existing long-term representations (pseudo-English words). Moreover, and perhaps most critically, the magnitude of the subsequent memory effect in pLIPC was reliably greater for pseudo-English than for English words, pointing to a differential role of phonological control in the building of memories for unknown phonological forms (Fig. 3). Thus, the processes subserved by pLIPC appear to be particularly important for later remembering when novel phonological representations must be constructed based on knowledge of orthographic-to-phonological mappings rather than when retrieval from long-term memory can provide the full representation. The prior failure to observe a reliable subsequent memory effect in pLIPC for English words [25] may reflect the fact that the magnitude of such effects is modest for known words, which have a facilitated and possibly direct route to phonology [13].

The present study also revealed an apparent difference between the magnitudes of the subsequent memory effect in pLIPC and those in parietal cortices, as well as the presence of functional differences between inferior and superior parietal structures. In contrast to the hypothesis that phonological storage processes are most critical for new word learning [2], the effect of word familiarity on the magnitude of the subsequent memory effect was reliable in pLIPC, but not in parietal cortices associated with the representation of phonological codes (Fig. 3). Bilateral superior parietal subsequent memory effects were numerically, but not statistically, greater for the pseudo-English than for the English words; this null effect of stimulus familiarity should be interpreted with caution. By contrast, the effect of familiarity on encoding responses in inferior parietal cortices clearly differed from that in pLIPC. Specifically, in contrast to pLIPC, the subsequent memory effects for pseudo-English and English words were comparable in left inferior parietal cortex, and subsequent memory correlates were not observed in right inferior parietal cortex (Fig. 3). These data differentially implicate the pLIPC-mediated control process in phonological assembly and word learning, and further point to functional heterogeneity within posterior parietal structures. Multiple parietal processes-perhaps inclusive of phonological representation and attentional switching mechanisms [23] — appear to be recruited during phonological analysis. Future studies may serve to further elucidate the nature of these mechanisms and their relation to word learning.

Given the above conceptual framework, a somewhat surprising outcome in the present study was the failure to observe greater pLIPC activation during Foreign, relative to pseudo-English, word processing. The pseudo-English stimuli had a higher similarity to the familiar English words than did the Foreign (Finnish) stimuli. That is, the statistical regularities (e.g. bigram frequencies) of the pseudo-English orthographic strings overlapped to a greater extent with English words as these strings were generated by changing one consonant in a base English word. Given this relation, one might have expected to observe greater pLIPC activation during phonological assembly of the Foreign than of the pseudo-English words. However, the fMRI results revealed the reverse pattern. There are several possible interpretations of this pattern. It may reflect the fact that the increased familiarity of the pseudo-English words, relative to Foreign words, was accompanied by an increased number of mappings to multiple known sublexical units. Moreover, a pre-existing representation from the lexicon could serve to constrain possible mappings for English words relative to novel words $[22,38]$. Alternatively, it is possible that a neighborhood of highly similar English words was activated as a set of possible matches for the pseudo-English stimulus [45]. In either case, although transformation of the Foreign strings to a phonological representation may have placed greater demands on pLIPC control processes, the pseudo-English words may have resulted in a greater load on the phonological system because multiple representations may have been brought on-line. Although speculative, to the extent that this was the case, then the greater pLIPC activation during pseudo-English relative to Foreign word processing may be akin to prior working memory load manipulations that have been observed to modulate activity in this region $[8,12]$.

Another intriguing aspect of the present results was the unexpected observation of activation in aLIPC during our phonological processing task (MNI coordinates of $-48,42$, 0 ; Talairach transformed coordinates of $-48,41,-2 ; \sim \mathrm{BA}$ 47/46/10). The focus of this response fell just anterior to the aLIPC region frequently implicated in controlled semantic retrieval $[6,9,19,21,30,36,46]$, whereby task-relevant semantic knowledge is recovered from long-term memory in the course of goal-directed behavior. In particular, this focus was anterior to previous foci associated with controlled semantic retrieval (MNI: $-51,21,-12$; [53]), semantic elaboration (MNI: $-45,30,-9$; [16]), episodic encoding during semantic elaboration (Talairach: $-50,28,12$; [54]), semantic relative to phonological analysis (Talairach: -37 , $28,-9$; [32]), selection demands during semantic retrieval (Talairach: $-45,4,30 ; 38,15,13$; and $-41,30,8$; [43]), 
and selection demands as revealed by semantic repetition priming (Talairach: $-44,-15,-22$; [44]). This region also was modestly anterior and ventral to a focus associated with semantic repetition priming (Talairach: $-43,34,12$; [49]), and slightly anterior to that observed during both semantic and phonological processing (Talairach: $-45,35,-4$; [22]). However, a similar anterior region was reported when contrasting semantic to phonological processing (Talairach: $-42,40,-8$; [32]), and during semantic repetition priming for words (Talairach: $-49,45,-2 ;$ [48]).

Recent data have raised questions regarding the nature of the selectivity of aLIPC computations. At present, it is unclear whether aLIPC function is strongly restricted to controlled semantic retrieval or whether aLIPC computations, while differentially mediating controlled semantic access, also may generalize to other feature domains. On the one hand, extensive evidence indicates that aLIPC is differentially engaged during semantic relative to phonological processing of words [22,25,32,34,37]. Moreover, Poldrack et al. reported greater aLIPC activation during syllable judgments for English than for pseudo-English words [32]. By contrast, a recent study of single-word processing using a one-back attentional paradigm revealed greater aLIPC activation during pseudo-English than English word processing [41]. Moreover, the present study, which used a similar syllable decision task to that of Poldrack and colleagues [32], revealed greater aLIPC activation during pseudo-English than English and Foreign words (Fig. 4). Although the source of the divergence between our results and those of Poldrack and colleagues remains unclear given the similarities between the two designs - again our aLIPC focus fell in a slightly more anterior/frontopolar region to that seen in the Poldrack study and in other investigations of controlled semantic retrieval-the unexpected, inverted-U shaped pattern of aLIPC activation across word types (Fig. 4) has implications for understanding the selectivity of aLIPC function.

One post hoc, and thus speculative, interpretation for this pattern of aLIPC activation is that it indicates that the functional contributions of this region may not be strongly restricted to semantic access [22]. Although pseudo-English words may make contact with multiple orthographically similar English words-thus likely resulting in activation of the corresponding semantic representations of these known entities [41] - a similar semantic retrieval account for the greater aLIPC activation observed during Foreign relative to English word processing seems problematic. An alternative interpretation, recently forwarded by Gold and colleagues [22], posits that aLIPC computations are engaged during conditions in which subjects are required to make a decision in under-constrained situations, as when multiple possible semantic or nonsemantic representations can be mapped to a stimulus. These authors observed that as the ambiguity between stimulus-representation mappings increased -operationalized as decreasing between-subject response agreement for a particular stimulus-so did activation in aLIPC. It is important to note that such ambiguity could emerge due to weaker stimulus-representation associations and/or due to the increased presence of alternative, competing representations.

Motivated by Gold's study, we implemented a similar analysis on the present data, but, in contrast to this recent report, failed to reveal a similar linear relation between the two measures (Fig. 4). While response consensus was lower for Foreign than for pseudo-English words, aLIPC activation was greater for the latter word type. What are the functional implications of this inverted-U pattern? Within the present context, aLIPC activation may be sensitive to the extent of possible matches between the orthographic input and stored phonological representations (at either a whole word or a sublexical level). As noted above, the number of possible matches would be greater for the pseudo-English than for the Foreign words, while pre-existing representations may constrain alternative matches for known English words. More broadly, these data, in conjunction with the extensive literature on the role of aLIPC in controlled semantic retrieval, raise the possibility that aLIPC mediates controlled access to target representations that are not automatically retrieved in response to bottom-up inputs. Moreover, these data, together with those of Gold and Buckner [22], raise the possibility that aLIPC-mediated controlled retrieval yields access to both abstract semantics and lexical knowledge. Further research is required to assess this post hoc interpretation; such studies will undoubtedly continue to clarify the functional contributions of aLIPC regions to word processing and memory formation.

Collectively, the present results suggest that the neural circuitry supporting phonological assembly and novel word learning includes bilateral parietal and LIPC structures. Posterior LIPC computations appear to play a central role in the building of novel phonological representations, illustrating the contributions of phonological control during word learning. These outcomes add to a growing literature pointing to interactions between multiple forms of memory [31,52], specifically highlighting a relation between cognitive control or working memory processes and episodic memory formation $[7,16,46]$.

\section{Acknowledgements}

Supported by the NIDCD (DC04466), NIMH (MH60941), National Science Foundation (0133126), McKnight Endowment Fund for Neuroscience, P. Newton, and a National Science Foundation Graduate Fellowship to DC. We thank Silvia Bunge and David Badre for insightful comments on an earlier draft of this manuscript, and Naomi Schmelzer and Matt Hutson for assistance with data collection.

\section{References}

[1] Awh E, Jonides J, Smith EE, Schumacher EH, Koeppe RA, Katz S. Dissociation of storage and rehearsal in verbal working 
memory: evidence from PET. Psychological Science 1996;7:2531.

[2] Baddeley A, Gathercole S, Papagno C. The phonological loop as a language learning device. Psychological Review 1998;105:158-73.

[3] Baddeley AD. Working memory. Oxford: Oxford University Press; 1986.

[4] Baddeley AD. Short-term phonological memory and long-term learning: a single-case study. European Journal of Cognitive Psychology 1993;5:129-48.

[5] Baddeley AD, Papagno C, Vallar G. When long term learning depends on short-term storage. Journal of Memory and Language 1988;27:586-95.

[6] Badre D, Wagner AD. Semantic retrieval, mnemonic control, and prefrontal cortex. Behavioral and Cognitive Neuroscience Reviews 2002;1:206-18.

[7] Braver TS, Barch DM, Kelley WM, Buckner RL, Cohen NJ, Miezin FM, et al. Direct comparison of prefrontal cortex regions engaged by working and long-term memory tasks. NeuroImage 2001;14:48-59.

[8] Braver TS, Cohen JD, Nystrom LE, Jonides J, Smith EE, Noll DC. A parametric study of prefrontal cortex involvement in human working memory. NeuroImage 1997;5:49-62.

[9] Buckner RL, Raichle ME, Petersen SE. Dissociation of human prefrontal cortical areas across different speech production tasks and gender groups. Journal of Neurophysiology 1995;74:2163-73.

[10] Caplan D. Language. Cambridge (MA): MIT Press; 1992.

[11] Cocosco CA, Kollokian V, Kwan RKS, Evans AC. Brainweb: Online interface to a 3D MRI simulated brain database. NeuroImage 1997;5:425.

[12] Cohen JD, Perlstein WM, Braver TS, Nystrom LE, Noll DC, Jonides $\mathrm{J}$, et al. Temporal dynamics of brain activation during a working memory task. Nature 1997;386:604-8.

[13] Coltheart M. The cognitive neuropsychology of reading. In: Posner MI, Marin OSM, editors. Attention and performance XI. Hillsdale (NJ): Erlbaum; 1985.

[14] D'Esposito M, Postle BR, Ballard D, Lease J. Maintenance versus manipulation of information held in working memory: An event-related fMRI study. Brain and Cognition 1999;41:66-86.

[15] Dale AM. Optimal experimental design for event-related fMRI. Human Brain Mapping 1999;8:109-14.

[16] Davachi L, Maril A, Wagner AD. When keeping in mind supports later bringing to mind: neural markers of phonological rehearsal predict subsequent remembering. Journal of Cognitive Neuroscience 2001;13:1059-70.

[17] Davachi L, Wagner AD. Hippocampal contributions to episodic encoding: insights from relational and item-based learning. Journal of Neurophysiology 2002;88:982-90.

[18] Demb J, Desmond JE, Wagner AD, Vaidya CJ, Glover GH, Gabrieli JDE. Semantic encoding and retrieval in the left inferior prefrontal cortex: a functional MRI study of task difficulty and process specificity. Journal of Neuroscience 1995;15:5870-8.

[19] Fiez JA. Phonology, semantics, and the role of the left inferior prefrontal cortex. Human Brain Mapping 1997;5:79-83.

[20] Fiez JA, Petersen SE. Neuroimaging studies of word reading. Proceedings of the National Academy of Sciences of United States of America 1998;95:914-21.

[21] Gabrieli JDE, Desmond JE, Demb JB, Wagner AD, Stone MV, Vaidya $\mathrm{CJ}$, et al. Functional magnetic resonance imaging of semantic memory processes in the frontal lobes. Psychological Science 1996;7:278-83.

[22] Gold BT, Buckner RL. Prefrontal cortex interacts with distinct posterior regions during controlled retrieval of semantic and phonological information. Neuron 2002;35:803-12.

[23] Jonides J, Schumacher EH, Smith EE, Koeppe RA, Awh E, Reuter-Lorenz PA, et al. The role of parietal cortex in verbal working memory. Journal of Neuroscience 1998;18:5026-34.

[24] Kucera H, Francis WN. Computational analysis of present-day English. Providence (RI): Brown University Press; 1967.
[25] Otten LJ, Rugg MD. Task-dependency of the neural correlates of episodic encoding as measured by fMRI. Cerebral Cortex 2001;11:1150-60.

[26] Paller KA, Wagner AD. Observing the transformation of experience into memory. Trends in Cognitive Science 2002;6:93-102.

[27] Papagno C, Valentine T, Baddeley AD. Phonological short-term memory and foreign-language vocabulary learning. Journal of Memory and Language 1991;30:331-47.

[28] Papagno C, Vallar G. Phonological short-term memory and the learning of novel words: the effect of phonological similarity and item length. Quarterly Journal of Experimental Psychology 1992;44A: 47-67.

[29] Paulesu E, Frith CD, Frackowiak RS. The neural correlates of the verbal component of working memory. Nature 1993;362:342-5.

[30] Petersen SE, Fox PT, Posner MI, Mintun M, Raichle ME. Positron emission tomographic studies of the cortical anatomy of single-word processing. Nature 1988;331:585-9.

[31] Poldrack RA, Clark J, Pare-Blagoev EJ, Shohamy D, Creso Moyano J, Myers C, et al. Interactive memory systems in the human brain. Nature 2001;414:546-50.

[32] Poldrack RA, Wagner AD, Prull MW, Desmond JE, Glover GH, Gabrieli JDE. Functional specialization for semantic and phonological processing in the left inferior frontal cortex. NeuroImage 1999;10:15-35

[33] Prabhakaran V, Narayanan K, Zhao Z, Gabrieli JD. Integration of diverse information in working memory within the frontal lobe Nature Neuroscience 2000;3:85-90.

[34] Price CJ, Moore CJ, Humphreys GW, Wise RSJ. Segregating semantic from phonological processes during reading. Journal of Cognitive Neuroscience 1997;9:727-33.

[35] Pugh KR, Shaywitz BA, Shaywitz SE, Constable RT, Skudlarski P, Fulbright RK, et al. Cerebral organization of component processes in reading. Brain 1996;119:1221-38.

[36] Raichle ME, Fiez JA, Videen TO, Macleod AMK, Pardo JV, Fox PT, et al. Practice-related changes in human brain functional anatomy during nonmotor learning. Cerebral Cortex 1994;4:8-26.

[37] Roskies AL, Fiez JA, Balota DA, Raichle ME, Petersen SE Task-dependent modulation of regions in the left inferior frontal cortex during semantic processing. Journal of Cognitive Neuroscience 2001;13:829-43.

[38] Seidenberg MS, McClelland JL. A distributed developmental model of visual word recognition and naming. Psychological Review 1989;96:523-68.

[39] Smith EE, Jonides J. Working memory in humans: neuropsychological evidence. In: Gazzaniga MS, editor. The cognitive neurosciences. Cambridge (MA): MIT Press; 1995.

[40] Smith EE, Jonides J. Storage and executive processes in the frontal lobes. Science 1999;283:1657-61.

[41] Tagamets M-A, Novick JM, Chalmers ML, Freidman RB. A parametric approach to orthographic processing in the brain: An fMRI study. Journal of Cognitive Neuroscience 2000;12:281-97.

[42] Talairach J, Tournoux P. A Co-planar stereotactic atlas of the human brain. Stuttgart: Thieme; 1988.

[43] Thompson-Schill SL, D'Esposito M, Aguirre GK, Farah MJ. Role of left inferior prefrontal cortex in retrieval of semantic knowledge: a reevaluation. Proceedings of the National Academy of Sciences of United States of America 1997;94:14792-7.

[44] Thompson-Schill SL, D'Esposito M, Kan IP. Effects of repetition and competition on activity in left prefrontal cortex during word generation. Neuron 1999;23:513-22.

[45] Vitevitch MS, Luce PA. Probabilistic phonotactics and neighborhood activation in spoken word recognition. Journal of Memory and Language 1999;40:374-408.

[46] Wagner AD. Working memory contributions to human learning and remembering. Neuron 1999;22:19-22.

[47] Wagner AD, Davachi L. Cognitive neuroscience: forgetting of things past. Current Biology 2001;11:R964-967. 
[48] Wagner AD, Desmond JE, Demb JB, Glover GH, Gabrieli JDE. Semantic repetition priming for verbal and pictorial knowledge: a functional MRI study of left inferior prefrontal cortex. Journal of Cognitive Neuroscience 1997;9:714-26.

[49] Wagner AD, Koutstaal W, Maril A, Schacter DL, Buckner RL. Task-specific repetition priming in left inferior prefrontal cortex. Cerebral Cortex 2000;10:1176-84.

[50] Wagner AD, Koutstaal W, Schacter DL. When encoding yields remembering: insights from event-related neuroimaging. Philosophical Transactions of the Royal Society of London Biology 1999;354:1307-24.

[51] Wagner AD, Maril A, Bjork RA, Schacter DL. Prefrontal contributions to executive control: fMRI evidence for functional distinctions within lateral prefrontal cortex. NeuroImage 2001;14:1337-47.
[52] Wagner AD, Maril A, Schacter DL. Interactions between forms of memory: when priming hinders new learning. Journal of Cognitive Neuroscience 2000;12(S2):52-60.

[53] Wagner AD, Paré-Blagoev EJ, Clark J, Poldrack RA. Recovering meaning: left prefrontal cortex guides controlled semantic retrieval. Neuron 2001;31:329-38.

[54] Wagner AD, Schacter DL, Rotte M, Koutstaal W, Maril A, Dale AM, et al. Building memories: remembering and forgetting of verbal experiences as predicted by brain activity. Science 1998;281:1188 91.

[55] Zatorre RJ, Meyer E, Gjedde A, Evans AC. PET studies of phonetic processing of speech: review, replication, and reanalysis. Cerebral Cortex 1996;6:21-30. 